DOI https://doi.org/10.30525/978-9934-26-046-9-30

\title{
HIGH-TEMPERATURE SUPERCONDUCTORS (HTSC) AS PROTECTIVE MATERIALS AGAINST THE EFFECTS OF ELECTROMAGNETIC RADIATION
}

\author{
Iasechko M. M. \\ Doctor of Technical Sciences, \\ Associate professor at the Department of Air Defense Armaments \\ of the Land Forces \\ Ivan Kozhedub Kharkiv National Air Force University \\ Mehelbei V. V. \\ Candidate of Technical Sciences, \\ Head of Research Laboratory \\ Ivan Kozhedub Kharkiv National Air Force University \\ Bologov A. \\ Department of Air Defense Armaments of the Land Forces \\ Ivan Kozhedub Kharkiv National Air Force University \\ Kharkiv, Ukraine
}

According to the results of [1-4], one of the ways to protect the REF (radio electronic facilities) from the effects of a powerful EMR through the antenna mechanism is protection based on strip-film structures, made out of high-temperature superconductors. Protection devices operate on the basis of the phase transitions use in the transmission lines of HTSCs, which use the transition from the superconducting state to the normally conducting state.

The widespread practical use of HTSC in microwave technology is primarily limited by their nonlinear properties and the need to use liquid nitrogen.

In [1], a device was considered to protect the receiver, which consists of a ferrite triple-shoulder wave guide Y-circulator, which switches to where the antenna is connected to the input of the first arm of the circulator, the absorbing load is connected to the output of the second arm, and the third arm is connected to the receiver. Inside the second arm, two parallel plates are installed, made out of a high-temperature superconductor, for example, of the $\mathrm{YBa} 2 \mathrm{Cu} 3 \mathrm{O} 7$ type with a thickness greater than the thickness of the 126 
skin layer in this superconductor. The space between the plates, which are adjacent to the walls of the wave guide shoulder, is filled with liquid nitrogen, coming through the pipes from the cryogenic generator of a closed cycle. The second shoulder 4 in the area of the plates 8 is placed inside the solenoid.

The device for protecting the receiver works as follows. First, the volume between the plates 8 is filled with liquid nitrogen, coming through the pipes from the cryogenic generator of a closed cycle.

A closed loop reduces the weight of the cooling system. Under the action of liquid nitrogen, the plates become superconducting. When lowpower signal carrying information arrives at the antenna, the signal, in accordance with the principle of the circulator, enters the other arm 4 . Since the plates are made out of HTSCs and are in superconducting states, the information signal is reflected from them and enters the third arm 6 and through it to the receiver. When the antenna receives high-power pulsed EMRs that exceed the power level of the transition of the superconducting plates to the normal state, this radiation enters the arm and destroys the superconductivity of the plates, transferring them into a nonsuperconducting (normal) state.

Thus, the receiver is protected automatically when the superconductivity of the plates is destroyed due to the energy of the very powerful radiation itself, and the transition time from the superconducting state to the normal state and vice versa is no more than 10-12 seconds. Adjustment of the protective power level is made by changing the magnitude of the magnetic field generated by the solenoid.

However, despite the simplicity of the protective device, the physical nature of the transition of the high-temperature superconductor to the normal state, under the influence of the microwave signal, has not yet been fully clarified.

The theoretical estimates show that already at power levels of the order of 10-3 W, a process of penetration into the HTSC film of Abrikosov's vortexes formed by a microwave field may occur, and therefore nonlinear effects appear and lower conductivity zones are formed.

This requires solving a number of problems related to the study of the basic properties of HTSC superconductors necessary for the creation of high-speed micro strip lines (MSL) and co planar lines (CPL) of transmission lines.

To implement the practical design of the protective device, it is necessary to conduct additional studies to optimize the design and topology, as 
well as the analysis of thermal processes at different amplitudes and duration of a parasitic EMR.

Nature-like technologies for creating materials and environmental protection environments.

In works $[1,3]$, it was proposed to use nature-like technologies that most satisfy the set of requirements for protection.

The use of nature-like (plasma) technologies introduces a number of new properties to the protection means compared to the conventional means. The most important of these are the instantaneous response of the state of the electronic subsystem, and accordingly, the change in the electrical properties of a solid-state and ionized air that determines the reflecting, absorbing and locking properties of a protective equipment under the influence of a powerful pulsed electromagnetic radiation EMR, performance throughout the life cycle of a REF, and the ability to withstand pulse over voltages.

A number of provisions of the general theory of radio absorbing materials and coatings, the theory of electrodynamics of composite materials, the kinetic theory of plasma, the fundamentals of electrodynamics of radiation and its interaction with matter, the theory of gas and plasma breakdown can be used in the study of individual properties of the plasma protective equipment. However, the analysis of the known scientific results in the study of the electrodynamics plasma medium characteristics showed the presence of a powerful pulsed EMR, a heterogeneous conductivity structure of the screen material due to the radioisotope and hexaferrite elements, the effect of changes in air pressure on the development of breakdown, the occurrence of Langmuir oscillations in the protective screen. This necessitates the development of a powerful pulsed ultra-wide band fields interaction theory with solid-state plasma-like materials and weakly ionized air medium. This will provide an opportunity to evaluate their response to the rate of rise of current and voltage, shielding, reflecting, absorbing and closing characteristics in order to determine the possibility of using plasma technologies to protect the REF electronic device from high-power pulsed electromagnetic radiation EMR without affecting their operation.

\section{References:}

1. Iasechko M., Tymochko O., Shapran Y., Trofymenko I., Maksiuta D., Sytnyk Y. Loss definition of charged particles in the discharge gap of the opening of the box-screens during the formation of a highly conductive channel. IJATCSE. 2019. № 8 (1.3). Pp. 1-9. 
2. Iasechko M., Larin V., Maksiuta D., Ochkurenko O., Samsonov Y., Lyashenko H., Zinchenko A. Influence of ionization source onto macroscopic parameters of the air media in the holes in cops-screensof radio electronic means. APRN Journal of Engineering and Applied Sciences. 2019. № 14 (20). Pp. 3566-3571.

3. Iasechko M., Turinskyi O., Burdin M., Larin V., Gnusov Y., Ikaev D., Borysenko V., Manoylo V. Protection of board radioelectronic equipment from the destructive powerful electromagnetic radiation with the use of natural technologies. IJETER. 2019. № 7 (11). Pp. 542-548.

4. Iasechko M., Sotnikov O., Syrotenko A., Larin V., Iasechko S., Ochkurenko O., Volkov A. Model of Combined Solid Plasma Material for the Protection of Radio-Electronic Means of Optical and Radio Radiation. IJATCSE. 2019. № 8 (4). pp. 1241 - 1247.

5. Iasechko M., Sotnikov O., Larin V., Ochkurenko O., Maksiuta D. The model of a medium for creation of electric hermetic screens of the radio electronic means. IJATCSE. 2019. 8(2). Pp. 300-304.

6. Iasechko M., Turinskyi O., Larin V., Prokopenko T., Kolmohorov O., Salash O., Tarshyn V. The Investigation of the Impulse Evolution of the Radio-Frequency and Optical Radiation During the Interaction With the Solid-State Plasma Media On Radioisotope and Hexaferrite Inclusions. IJETER. 2020. 8(4). Pp. 1333-1337. 